\title{
Echocardiographic markers of cardiac amyloidosis in patients with heart failure and left ventricular hypertrophy
}

\author{
Jorge Melero Polo ${ }^{1}$, Ana Roteta Unceta Barrenechea ${ }^{2}$, Pablo Revilla Martí1, \\ Raquel Pérez Palacios ${ }^{3}$, Anyuli Gracia Gutiérrez ${ }^{4}$, Esperanza Bueno Juana ${ }^{4}$, \\ Alejandro Andrés Gracia ${ }^{2}$, Saida Atienza Ayala ${ }^{5}$, Miguel Ángel Aibar Arregui ${ }^{4}$ \\ ${ }^{1}$ Cardiology Department, Hospital Clínico Universitario Lozano Blesa, Zaragoza, Spain \\ ${ }^{2}$ Nuclear Medicine Department, Hospital Clínico Universitario Lozano Blesa, Zaragoza, Spain \\ ${ }^{3}$ Biochemistry and Molecular Biology, IIS-Aragón, Zaragoza, Spain \\ ${ }^{4}$ Internal Medicine Department, Hospital Clínico Universitario Lozano Blesa, Zaragoza, Spain \\ ${ }^{5}$ Neurology Department, Hospital Clínico Universitario Lozano Blesa, Zaragoza, Spain
}

\begin{abstract}
Background: Cardiac amyloidosis (CA), following a non-invasive diagnosis, constitutes an increasingly prevalent heart failure (HF) etiology. This study aims to determine which echocardiography findings help to diagnose CA in patients with left ventricular hypertrophy (LVH) admitted for decompensated HF.

Methods: The present study is a retrospective observational study on a cohort of $85 \mathrm{LVH}$ patients admitted for HF decompensation, in which ${ }^{99 m} T c-D P D$ scanning was performed to rule out transthyretin $C A$. The echocardiographic findings obtained were compared between CA and non-CA groups.

Results: From a total number of 85 patients, 49 (57.6\%) met the CA criteria and 36 (42.3\%) were ruled out for the disease. Interventricular septum thickness ( $16 \pm 3 \mathrm{~mm}$ vs. $14 \pm 3 \mathrm{~mm}$ ), left ventricular posterior wall thickness ( $14 \pm 3 \mathrm{~mm} v \mathrm{vs} .11 \pm 2 \mathrm{~mm}$ ), left ventricular mass $(259 \pm 76 \mathrm{~g} v \mathrm{~s} .224 \pm 53 \mathrm{~g}$ ), left ventricular end-diastolic diameter $(48 \pm 7 \mathrm{~mm}$ vs. $53 \pm 6 \mathrm{~mm}$ ), left ventricular end-diastolic indexed volume $\left(51 \pm 18 \mathrm{~cm}^{3} / \mathrm{m}^{2}\right.$ vs. $\left.59 \pm 16 \mathrm{~cm}^{3} / \mathrm{m}^{2}\right)$, tricuspid annular plane systolic excursion (16 \pm $\pm 5 \mathrm{~mm}$ vs. $20 \pm 4 \mathrm{~mm})$, right atrial area $\left(27.4 \pm 8.4 \mathrm{~cm}^{2}\right.$ vs. $\left.22.2 \pm 5.7 \mathrm{~cm}^{2}\right)$ and strain relative apical sparing $(2.2 \pm 0.9$ vs. $1.03 \pm 0.4 ; p=0.04)$ were significantly associated with the diagnosis of $C A$. Conclusions: In patients with LVH admitted for HF decompensation, there are several echocardiographic features (LVH, reduced left ventricular cavity size, strain relative apical sparing, right atrial dilation, and altered right ventricular function) that are associated with the diagnosis of cardiac amyloidosis. (Cardiol J 2023; 30, 2: 266-275)
\end{abstract}

Key words: cardiac amyloidosis, echocardiography, heart failure, left ventricular hypertrophy

\section{Introduction}

Cardiac amyloidosis (CA) is an infiltrative disease caused by the extracellular deposition of defective proteins. To date, more than 30 poten- tially amyloidogenic proteins have been described, although the majority of amyloidosis with cardiac presentation that are present in the clinical practice are systemic immunoglobulin light chain (AL) and transthyretin amyloidosis (ATTR), which, in turn,

Address for correspondence: Dr. Jorge Melero Polo, Cardiology Department, Hospital Clínico Lozano Blesa de Zaragoza, calle San Juan Bosco 15, CP: 50009 Zaragoza, Spain, tel: +34 6610585 66, e-mail: jorgemeleropolo@gmail.com 
is divided into the hereditary or variant (ATTR-v) and the senile or wild-type (ATTR-wt) forms.

Cardiac amyloidosis diagnosis required, until recent years, proof of amyloid material recovered by biopsy. Recently, a cardiac ATTR non--invasive diagnostic by gammagraphy with specific radiotracers and exclusion of the monoclonal component has been validated [1], and since, more cases, mainly from the wild type subtype, have been diagnosed without the need for histology. In fact, employing this approach, in recent reviews and scientific statements recommended histology just in two scenarios: a grade 1 uptake of myocardial radiotracer on cardiac gammagraphy or the presence of a monoclonal protein [2, 3].

The echocardiogram is often the first test that allows suspicion to the diagnosis of CA. Among all the characteristic echocardiographic findings of CA, the most frequent is left ventricular (LV) thickening [4]. This finding is a good starting point for establishing the suspicion of CA, but it is a non-specific finding that can be objectified by a multitude of causes: arterial hypertension, aortic valve disease, some myocardial disease or simply due to intensive training [5]. For all these reasons, some studies have sought new echocardiographic parameters in order to distinguish patients with amyloidosis from others with LV thickening of a different origin, especially arterial hypertension and hypertrophic cardiomyopathy [6-9]. To date however, none of the published studies have compared the echocardiographic characteristics of patients hospitalized for heart failure (HF) with final diagnosis of CA versus those with LV thickening of another cause.

Some research groups have found an ATTR prevalence of up to $13 \%$ in patients $>60$ years old admitted for $\mathrm{HF}$ with preserved ejection fraction and left ventricular hypertrophy (LVH) [4]. Thus, it would be extremely useful to identify which echocardiographic parameters allow us to increase the diagnostic probability of CA in this subgroup, and hence select the patients that could benefit from a cardiac gammagraphy.

The aim of the study was to compare the echocardiographic findings from patients hospitalized due to CA and from patients with $\mathrm{HF}$ and $\mathrm{LVH}$ of other origins.

\section{Methods}

\section{Study population}

The study population consisted of a consecutive series of patients hospitalized in the document- ed center between November 2013 and December 2019, due to de novo or chronic uncompensated $\mathrm{HF}$, who presented LVH and who subsequently underwent a gammagraphy with ${ }^{99 \mathrm{~m}} \mathrm{Tc}-\mathrm{DPD}$ to rule out CA by transthyretin. The study cases selected were those patients who finally met the diagnostic criteria of CA, and for controls by those who did not meet the criteria; controls were paired by sex and age to avoid bias against study cases (Fig. 1).

Inclusion criteria: Patients $>18$ years of age, with an established diagnosis of $\mathrm{HF}$ [8] and LVH by echocardiogram, who underwent a DPD gammagraphy during a de novo $\mathrm{HF}$ admission or known HF decompensation.

Exclusion criteria: Failure to meet any of the inclusion criteria. Refusal to participate in the study.

\section{Sample size}

Given that the number of cases with a CA diagnosis in the selected environment was limited, and with the purpose of increasing the statistical power of the study, all patients who met the inclusion criteria were selected through a sampling of consecutive cases.

\section{Diagnostic criteria}

For the diagnosis of transthyretin cardiac amyloidosis, we relied on the criteria as defined by the study of Gillmore et al. [1]: a grade 2-3 uptake of the myocardial radiotracer on cardiac gammagraphy with ${ }^{99 \mathrm{~m}} \mathrm{Tc}-\mathrm{DPD}$ presents a sensitivity of $91 \%$ and a specificity of $87 \%$ for the diagnosis of ATTR; this specificity increases to $100 \%$ (with respect to an invasive diagnosis by endomyocardial biopsy) by adding the criteria of absence of monoclonal protein in blood and urine. In the present study, since immunofixation was not performed in every patient during their hospitalization, the diagnosis of ATTR with grade 2-3 of gammagraphic capture in addition to a negative immunofixation or absence of diagnosis of monoclonal gammopathy in the monitoring was used. Considering this, although we can strongly state that the vast majority of the cardiac amyloidoses corresponded to the TTR subtype, it cannot be ruled out that some cases may correspond to other subtypes, therefore we did not distinguish between the different subtypes of amyloidosis and refer to this group as the group with CA. The study patients with a grade 1 uptake of the myocardial radiotracer or patients with monoclonal protein were excluded.

Left ventricular hypertrophy was defined as a thickness greater than or equal to $12 \mathrm{~mm}$ in any 


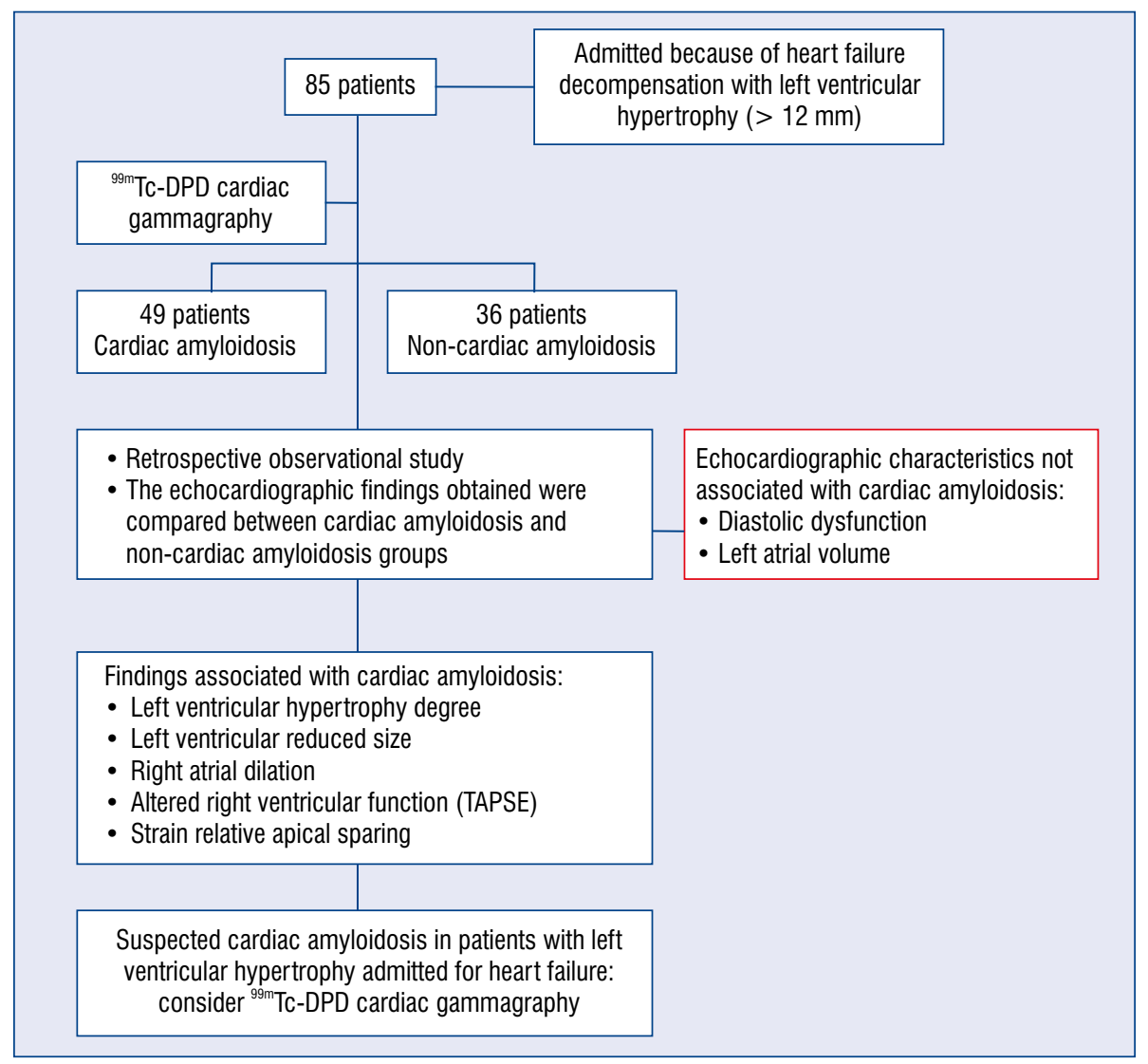

Figure 1. The present study showed that in patients with left ventricular hypertrophy admitted for heart failure decompensation, several echocardiographic features are associated with the diagnosis of cardiac amyloidosis; TAPSE - tricuspid annular plane systolic excursion.

segment of the LV wall as measured by echocardiogram.

For the diagnosis of HF, the diagnostic criteria described in the latest European Society of Cardiology (ESC) guidelines were used [10].

\section{Clinical, analytical and echocardiographic collected data}

For this study, the following parameters were collected:

- Demographic variables: sex and age;

- Relevant background: cardiovascular risk factors, evidences of atrial fibrillation or ischemic heart disease;

- Basal New York Heart Association class;

- Analytical parameters determined in the same admission in which the gammagraphy-DPD was requested: N-terminal pro B-type natriuretic peptide (NT-proBNP), ultra-sensitive troponin T, and glomerular filtering (CKD-EPI equation, expressed in $\mathrm{mL} / \mathrm{min} / 1.73 \mathrm{~m}^{2}$ );

- Echocardiographic data: echocardiograms were performed in cabinet echocardiograms, in Siemens Acuson sc2000 and Philips Envisor HD echocardiographs, by experienced independent echocardiographers. The measurements were obtained at the time of admission, without yet knowing the patient's diagnosis, thus avoiding potential biases. Interventricular septum and LV posterior wall maximum thickness, telediastolic and telesystolic LV diameters, LV mass, right atrial area, right ventricle basal diameter and left atrial volume were determined in two-dimensions. The Simpson biplanar method was used to calculate ejection fraction. Diastolic function was evaluated according to the current recommendations, by analyzing the transmitral flow by pulsed Doppler and mitral annulus speed by tissue Doppler, evaluating both E/A and E/e' ratio; pulmonary artery systolic pressure was estimated through tricuspid regurgitation; right ventricular function was evaluated by tricuspid annular plane systolic excursion (TAPSE) and S' measurements. The presence of pericardial effusion was also evaluated. During the echo- 


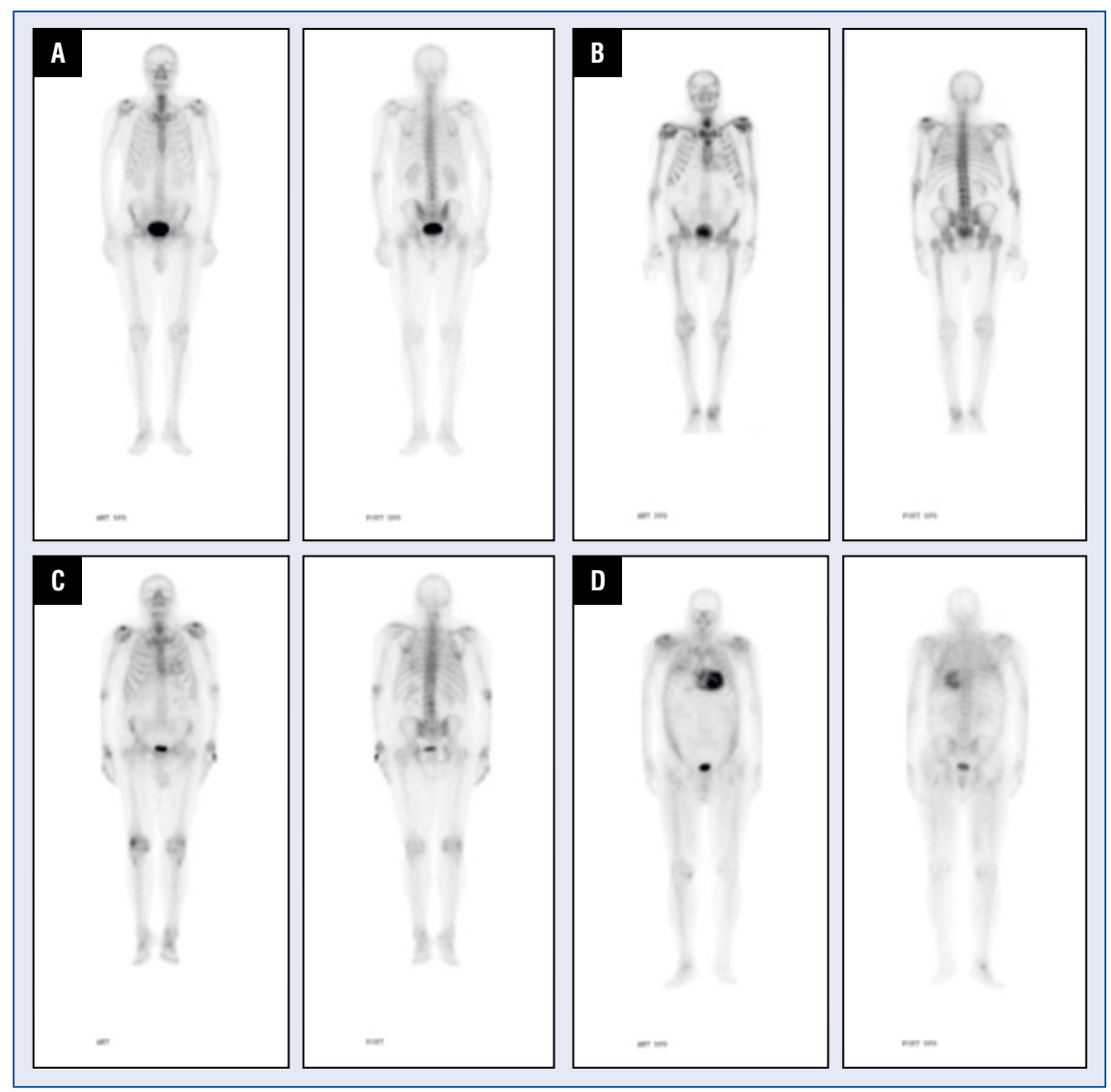

Figure 2. Cardiac gammagraphies with ${ }^{99 \mathrm{~m}} \mathrm{Tc}-\mathrm{DPD}$, with different uptake grades in Perugini score; A. Grade 0; B. Grade 1; C. Grade 2; D. Grade 3.

cardiogram, both heart rate and body surface were recorded. All measurements were performed according to ESC guidelines [11];

- Gammagraphic data: the degree of gammagraphic uptake was collected in the test, according to which the diagnosis of cardiac amyloidosis by TTR was either confirmed or excluded. The degree of intensity of the radiotracer uptake using the Perugini scale (Fig. 2) [12].

\section{Ethical aspects}

The study was conducted in full compliance with current ethical standards (Declaration of Helsinki); an informed consent was obtained for each patient's data processing. The study was approved by our center's Ethics Committee. All data were collected in a secure database, guaranteeing total confidentiality.

\section{Statistical analysis}

Statistical analysis was performed with IBM SPSS Statistics version 22. Variables were expressed as mean \pm standard deviation. Categorical variables are expressed as \%. To evaluate the differences between these categorical variables, the $\chi^{2}$ test and the Fisher test were used, as appropriate in each case. For continuous variables with normal distribution, the Student $t$ test was used for independent samples. A p-value $<0.05$ was considered statistically significant.

\section{Results}

\section{Characteristics of the patients}

Eighty-five patients were included in the study, with a mean age of $80 \pm 9$ years and from which $75.3 \%$ were male. $49(57.6 \%)$ patients met the diagnostic criteria for CA (ATTR group) and in 36 (42.3\%) patients the disease was ruled out (non-ATTR group).

Sex distribution was similar in both groups (77.6\% vs. $72.2 \%$; $=0.57)$. In terms of age, patients with ATTR were slightly older ( $83 \pm 9$ vs. $77 \pm 8 ; \mathrm{p}=0.003)$. There were no significant differences between both groups in atrial fibrillation rate, heart rate or body surface (Table 1).

Patients in the control group presented a higher rate of cardiovascular risk factors, which was only statistically significant in the case of dia- 
Table 1. Basal data.

\begin{tabular}{lcccc}
\hline & Total & Amyloidosis & Non-amyloidosis & P \\
\hline Total number of patients & 85 & 49 & 36 \\
Male & $64(75.3 \%)$ & $38(77.6 \%)$ & $26(72.2 \%)$ & 0.57 \\
Age [years] & $80 \pm 9$ & $83 \pm 9$ & $77 \pm 8$ & $0.003^{*}$ \\
Arterial hypertension & $73(85.9 \%)$ & $39(71.6 \%)$ & $34(94.4 \%)$ & 0.105 \\
Diabetes mellitus & $32(37.6 \%)$ & $12(24.5 \%)$ & $20(55.6 \%)$ & $0,03^{*}$ \\
Dyslipidemia & $52(61.2 \%)$ & $26(53.1 \%)$ & $26(72.2 \%)$ & $0.092^{*}$ \\
Glomerular filtration & $48 \pm 19$ & $48.4 \pm 19$ & $48 \pm 19$ & 0.88 \\
Ischemic cardiomyopathy & $21(24.7 \%)$ & $10(20.4 \%)$ & $11(30.6 \%)$ & 0.28 \\
B-type natriuretic peptide $[\mathrm{pg} / \mathrm{mL}]$ & $10069 \pm 12717$ & $10500 \pm 13800$ & $9500 \pm 11500$ & 0.72 \\
Troponin T [pg/mL] & $128 \pm 219$ & $180 \pm 272$ & $50 \pm 27$ & $0.041^{*}$ \\
\hline
\end{tabular}

*Sex distribution was similar in both groups. Patients with transthyretin amyloidosis were slightly older. Patients in the control group presented a higher rate of cardiovascular risk factors.

betes mellitus ( $56 \%$ vs. $25 \%$; $\mathrm{p}=0.03$ ), although showing a trend in arterial hypertension (94\% vs. $71 \% ; \mathrm{p}=0.105)$, and dyslipemia ( $72 \%$ vs. $53 \%$; $\mathrm{p}=0.092)$. Ischemic heart disease rate was also slightly higher in the control group (30\% vs. $20 \%$; $\mathrm{p}=0.28)$.

\section{Biomarkers}

Troponin records on admission were significantly higher in patients with amyloidosis than in controls $(180 \pm 272$ vs. $50 \pm 27 ; \mathrm{p}=0.041)$. However, no significant differences were found in NT-proBNP and glomerular filtration values between groups.

\section{Echocardiographic parameters}

Interventricular septum thickness $(16 \pm 3 \mathrm{~mm}$ vs. $14 \pm 3 \mathrm{~mm} ; \mathrm{p}=0.002)$ and $\mathrm{LV}$ posterior wall thickness $(14 \pm 3 \mathrm{~mm}$ vs. $11 \pm 2 \mathrm{~mm} ; \mathrm{p}<0.001)$ were significantly higher in the CA group. LV mass, both as absolute values ( $259 \pm 76 \mathrm{~g}$ vs. $224 \pm 53 \mathrm{~g}$; $\mathrm{p}=0.026)$ and as indexed $\left(151 \pm 43 \mathrm{~g} / \mathrm{m}^{2}\right.$ vs. $\left.123 \pm 23 \mathrm{~g} / \mathrm{m}^{2} ; \mathrm{p}=0.002\right)$, was also significantly higher in the ATTR group.

Regarding LV dimensions, both telediastolic diameter ( $48 \pm 7 \mathrm{~mm}$ vs. $53 \pm 6 \mathrm{~mm} ; \mathrm{p}=0.004)$ and indexed telediastolic volume $\left(51 \pm 18 \mathrm{~cm}^{3} / \mathrm{m}^{2}\right.$ vs. $59 \pm 16 \mathrm{~cm}^{3} / \mathrm{m}^{2} ; \mathrm{p}=0.004$ ) were significantly smaller in amyloidosis group. Telesystolic diameter and indexed telesystolic volume did not show statistically significant differences.

Strain data were recorded in only 15 patients. Relative apical sparing was significantly higher in the CA group $(2.2 \pm 0.9$ vs. $1.03 \pm 0.4 ; \mathrm{p}=0.04)$. Global longitudinal strain showed a clear trend towards its reduction in patients with CA $(-12 \pm$ $\pm 5.5 \%$ vs. $-19 \pm 0.6 \% ; p=0.078)$, although differences were not statistically significant.

Indexed left atrial volume was very high in both groups ( $55 \pm 17 \mathrm{~cm}^{3}$ vs. $51 \pm 17 \mathrm{~cm}^{3} ; \mathrm{p}=0.5$ ), in the range of severe left atrial dilatation. No statistically significant differences were found.

Regarding right cavities, both right atrial area $\left(27.4 \pm 8.4 \mathrm{~cm}^{2}\right.$ vs. $\left.22.2 \pm 5.7 \mathrm{~cm}^{2} ; \mathrm{p}=0.04\right)$ and TAPSE $(16 \pm 5 \mathrm{~mm}$ vs. $20 \pm 4 \mathrm{~mm} ; \mathrm{p}=0.004)$ were more affected in the amyloidosis group. No significant differences were found in either the S'-wave, or the estimation of the pulmonary artery systolic pressure, or the right ventricle basal diameter.

Finally, regarding diastolic function parameters (quotients E/a and E/e') although a trend towards greater involvement was observed in patients with CA, no statistically significant differences were found. All results are shown in Tables 1 and 2 .

\section{Discussion}

Cardiac amyloidosis is increasingly relevant for several reasons: Non-invasive diagnosis [1] was proved to be in higher prevalence of ATTR. In addition, recent studies with new drugs [13-15] have shown therapeutic targets capable of modifying the natural history of the disease. This highlights the importance of early diagnosis in an entity still underdiagnosed [16], among other reasons because it presents clinical manifestations that are similar to other much more frequent heart diseases [17].

The first test that often leads to CA clinical suspicion is the echocardiogram [18]. The most common echocardiographic findings in these patients are interventricular septum thickening and 
Table 2. Echocardiographic data.

\begin{tabular}{|c|c|c|c|c|}
\hline & Total & Amyloidosis & $\begin{array}{l}\text { Other heart failure } \\
\text { (wall > } 12 \mathrm{~mm} \text { ) }\end{array}$ & $\mathbf{P}$ \\
\hline Interventricular septum [mm] & $15 \pm 3$ & $16 \pm 3$ & $14 \pm 3$ & $0.002^{*}$ \\
\hline LV posterior wall [mm] & $13 \pm 3$ & $14 \pm 3$ & $11 \pm 2$ & $<0.001^{*}$ \\
\hline LVEDD [mm] & $50 \pm 7$ & $48 \pm 7$ & $53 \pm 6$ & $0.004 *$ \\
\hline LVESD [mm] & $35 \pm 7$ & $35 \pm 7$ & $35 \pm 6$ & 0.086 \\
\hline LVEDVI $\left[\mathrm{cm}^{3} / \mathrm{m}^{2}\right]$ & $55 \pm 17$ & $51 \pm 18$ & $59 \pm 16$ & $0.004^{*}$ \\
\hline LVESVI $\left[\mathrm{cm}^{3} / \mathrm{m}^{2}\right]$ & $26 \pm 11$ & $25 \pm 11$ & $27 \pm 12$ & 0.49 \\
\hline LV mass $[\mathrm{g}]$ & $243 \pm 68$ & $259 \pm 76$ & $224 \pm 53$ & $0.026^{*}$ \\
\hline Indexed mass $\left[\mathrm{g} / \mathrm{m}^{2}\right]$ & $138 \pm 38$ & $151 \pm 43$ & $123 \pm 23$ & $0.002^{*}$ \\
\hline LA volume $\left[\mathrm{cm}^{3}\right]$ & $53 \pm 17$ & $55 \pm 17$ & $51 \pm 17$ & 0.5 \\
\hline $\mathrm{RA}$ area $\left[\mathrm{cm}^{2}\right]$ & $25 \pm 8$ & $27.4 \pm 8.4$ & $22.2 \pm 5.7$ & $0.04^{*}$ \\
\hline RV basal diameter [mm] & $40 \pm 7$ & $40 \pm 9$ & $40 \pm 5$ & 0,87 \\
\hline $\mathrm{E} / \mathrm{A}$ ratio & $1.4 \pm 0.9$ & $1.7 \pm 1$ & $1 \pm 0.4$ & 0.062 \\
\hline E/e' ratio & $17.3 \pm 7.1$ & $18.1 \pm 7.2$ & $16.4 \pm 7$ & 0.36 \\
\hline Relative apical sparing & $1.7 \pm 0.8$ & $2.2 \pm 0.9$ & $1.03 \pm 0.4$ & 0.04 \\
\hline Global longitudinal strain [\%] & & $-12 \pm 5.5$ & $-19 \pm 0.7$ & 0.078 \\
\hline TAPSE [mm] & $18 \pm 5$ & $16 \pm 5$ & $20 \pm 4$ & $0.004 *$ \\
\hline $\mathrm{S}^{\prime}$ wave RV & $11.4 \pm 4$ & $11.2 \pm 4.7$ & $11.7 \pm 2.1$ & 0.7 \\
\hline PASP [mmHg] & $44 \pm 15$ & $44 \pm 15$ & $44 \pm 14$ & 0.96 \\
\hline LV ejection fraction [\%] & $54 \pm 10$ & $52 \pm 10$ & $55 \pm 12$ & 0.18 \\
\hline Effusion presence & $14(17 \%)$ & $9(23.7 \%)$ & $5(14.3 \%)$ & 0.56 \\
\hline
\end{tabular}

*Interventricular septum thickness, left ventricular posterior wall thickness, left ventricular mass, left ventricular end-diastolic diameter, left ventricular end-diastolic indexed volume, tricuspid annular plane systolic excursion, right atrial area and strain relative apical sparing were significantly associated with the diagnosis of cardiac amyloidosis. LV — left ventricle; LA — left atrium; RV - right ventricle; RA - right atrium; LVEDD - left ventricular end-diastolic diameter; LVESD - left ventricular end-systolic diameter; LVEDVI — left ventricular end-diastolic volume index; LVESVI - left ventricular end-systolic volume index; TAPSE — tricuspid annular plane systolic excursion; PASP — pulmonary artery systolic pressure

biauricular dilation. Diastolic dysfunction, with restrictive transmitral flow and increased filling pressures, is also a frequent finding with reduced e' wave from early stages of the disease. However, as these alterations are also found in many patients with HF, in many cases they do not assist in suspecting CA as an etiological diagnosis [19]. The speckled myocardial appearance (which has the disadvantage of being subjective and therefore highly operator dependent) and valve thickenings, which are more specific parameters, do not usually appear until advanced stages of the disease, and therefore are not reliable parameters for early diagnosis [20]. For all these reasons, it seems increasingly important to find parameters that help to select the patients most likely to suffer CA, in order to choose the candidates for new diagnostic tests, thus, being vital to study the echocardiographic characteristics of these patients.

The present study revealed that a greater LV wall thickness and smaller LV cavity size (meas- ured by both diameter and telediastolic volume) may help to discriminate CA patients from patients with $\mathrm{HF}$ and LVH. This data is consistent with results in Uzan et al. [6], although in their study controls are formed by patients with LVH without HF. Also, Boldrini et al. [21], in a recent article included the LV wall thickness in a diagnostic score of CA in patients with LVH and confirm the diagnostic value of the wall thickness and the LV mass in these patients (Fig. 3).

The value of different parameters of the right ventricle cavities in the echocardiographic diagnosis of CA has been previously shown [6]: both the parameters of right ventricular systolic function (measured as TAPSE, S' and fractional area), and the size of the right atrium and right ventricle were significant in this regard, being the right ventricular free wall longitudinal strain (RV FWLS) the parameter with the greatest power to discriminate CA from other pathologies presenting hypertrophy. Other studies have already shown 


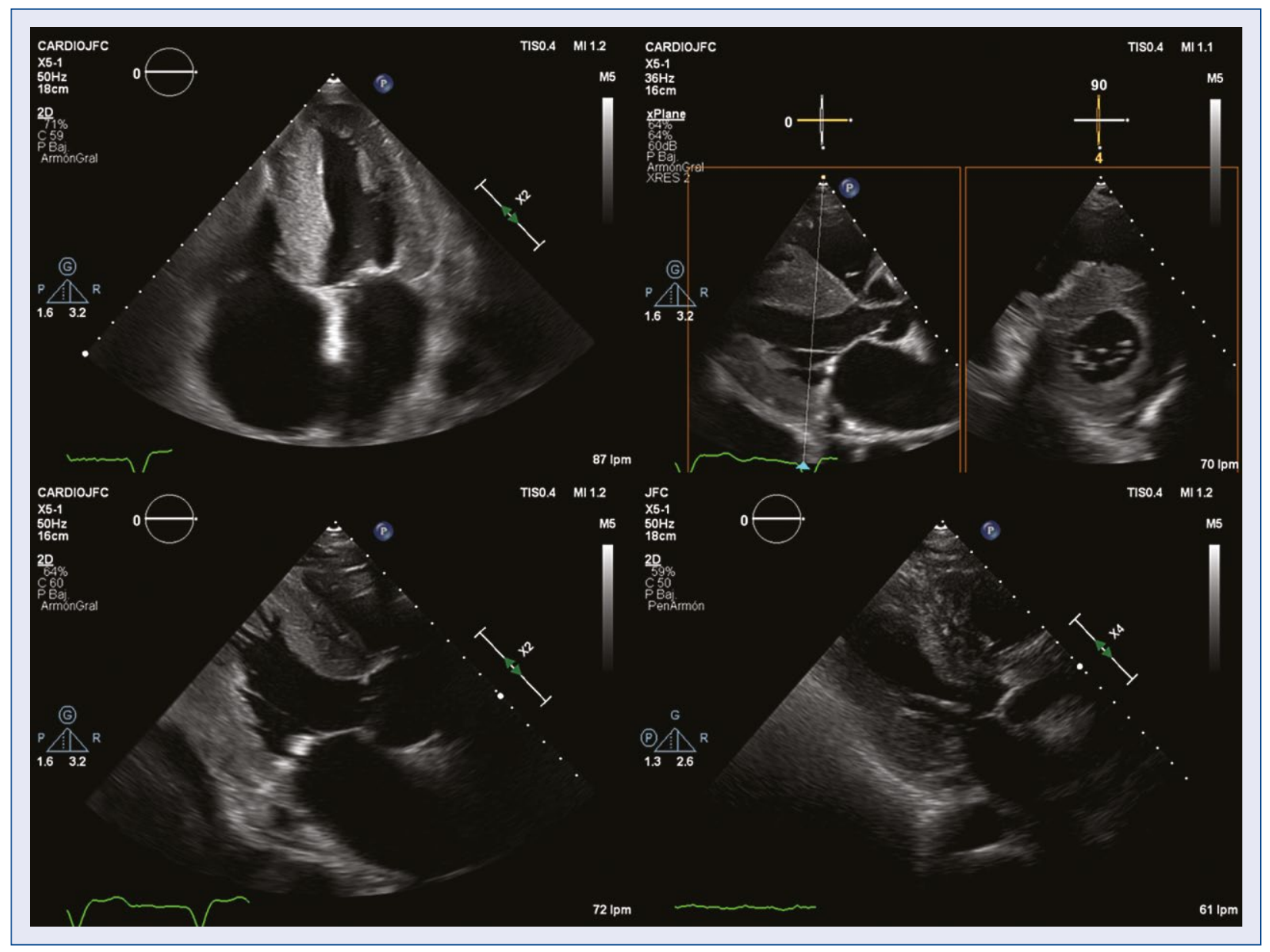

Figure 3. Echocardiography. The degree of left ventricular hypertrophy, left ventricular reduced size and right atrial dilatation increase the suspicion of cardiac amyloidosis.

the systolic involvement of the right ventricle measured by TAPSE or the fractional area in comparison with controls with LVH, patients with amyloidosis without cardiac involvement or healthy controls [21-23], as well as the prognostic value of such manifestation [22-24]. In the current study, in which the control group consisted of patients with HF and LVH, the differences in TAPSE and right atrial area were equally significant, which seems to reinforce the hypothesis that the involvement of the right cavities is an echocardiographic finding in favor of the diagnosis of CA. Similar to other studies that have studied right ventricular function in patients with CA, in the present study no difference was found in the pulmonary artery systolic pressure estimated through tricuspid regurgitation, which reinforces the idea that the right dysfunction is due to amyloid infiltration (as occurs in other cardiac structures) and not only to increased preload.
Regarding those values with no statistical significance in the current sample, perhaps the most striking is the diastolic dysfunction, which in the present study was measured from the E/A and $\mathrm{E} / \mathrm{e}$ ' ratios. In several studies $[7,21]$ comparing patients with CA versus $\mathrm{LVH}$ from another origin, statistically significant differences were obtained for this parameter; the substantial difference lies in the fact that the current controls also have HF (unlike the majority of studies with hypertensive controls or with hypertrophic cardiomyopathy without $\mathrm{HF}$ ) having therefore a greater degree of diastolic dysfunction, with a much-increased average filling pressure in both groups. Moreover, the fact that they are patients admitted for HF provokes that the filling pressures of all these patients are even more increased. Thus, although the diastolic dysfunction is a typical feature in an early-stage of CA, it does not appear to be as discriminating as other parameters in a cohort of $\mathrm{HF}$ and $\mathrm{LVH}$ patients. 


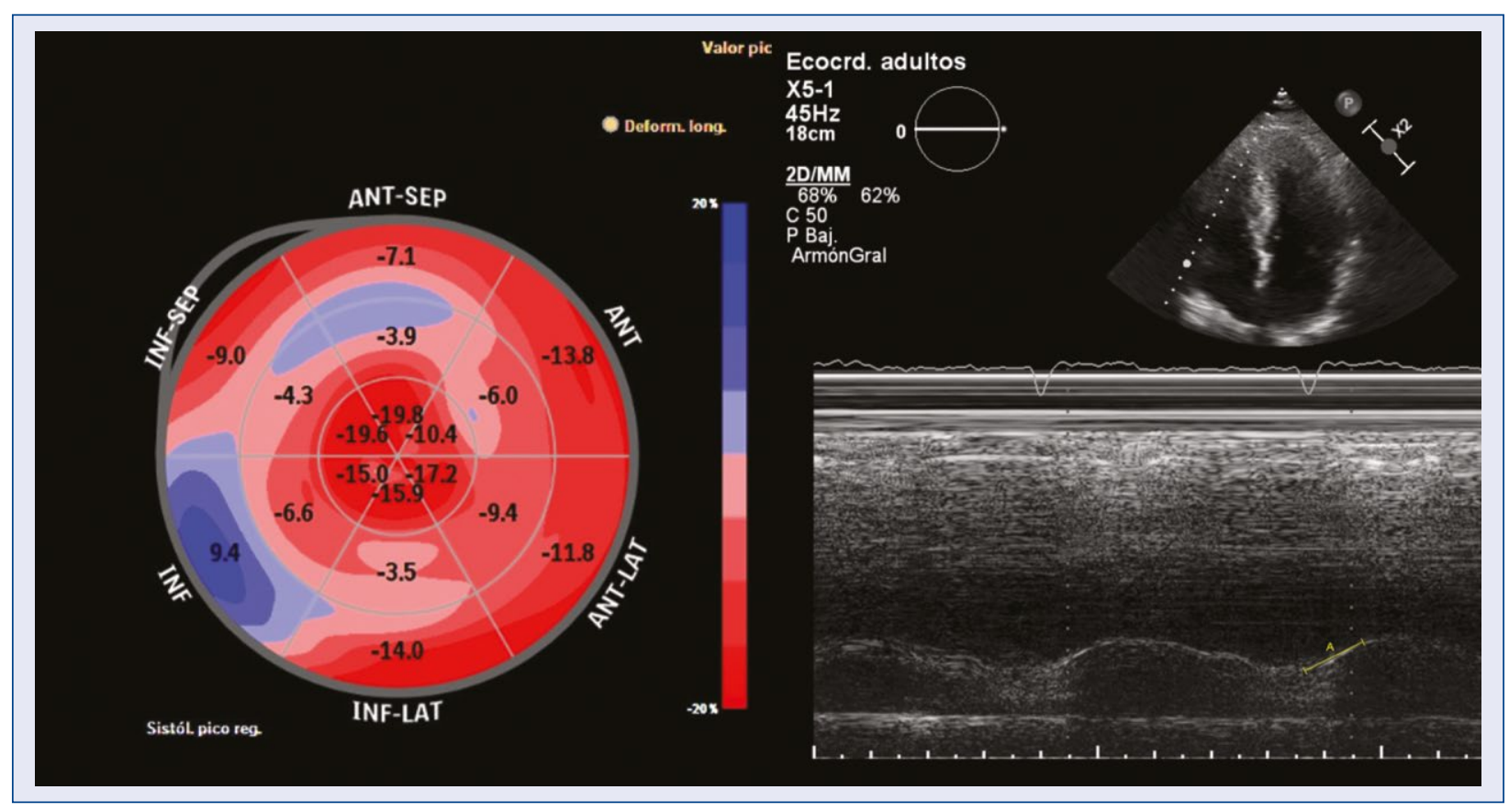

Figure 4. Altered right ventricular function and strain relative apical sparing also increase the suspicion of cardiac amyloidosis.

Something similar occurs with the LV ejection fraction [21] and left atrial volume, which in several studies [7,25] have been shown to discriminate CA versus hypertensive patients or patients with hypertrophic cardiomyopathy. In one of these studies [25], the left atrium in patients with CA was in the range of severe dilation (similar to the present study) but the control group was composed of hypertensive patients, with much less dilated atria. In the current study, both groups presented means of severe dilation of the left atrium: when compared to controls with HF, this parameter is not as useful to differentiate patients with cardiac amyloidosis. Thus, the results in Liu et al. [7], in which the indexed left atrial volume showed high specificity to confirm the diagnosis of CA versus others with hypertrophic cardiomyopathy or hypertensive heart disease, were not confirmed in the present work versus controls with HF.

A parameter that has been widely studied in echocardiogram diagnosis, and which diagnostic value has been confirmed, is the involvement of the global longitudinal strain with preserved apical segment strain $[6,7,21,26,27]$. In the current series, despite strain data was only available in a few cases, relative apical sparing reached statistical significance, in accordance with the literature. Global longitudinal strain also showed a tendency to be clearly more impaired in patients with amyloidosis (Fig. 4).
Since this is a retrospective observational study with a relatively small sample size, specific cut off values were not recommended for each one of these echocardiograhic parameters. However, given the high prevalence of the disease in this subgroup of patients (up to 13\%) the authors would recommend that if severe right atrial dilatation, altered right ventricular function or relative apical sparing were found in patients with $\mathrm{HF}$ and $\mathrm{LVH}$, gammagraphy should be mandatory.

Regarding biomarkers, the diagnostic value of the troponin to discriminate patients with CA compared to patients with LVH had already been highlighted in some studies [28]. The work herein, also confirms this point when compared to controls with $\mathrm{HF}$.

The present study presents several limitations. First, the fact that only about half of the patients with CA have immunofixation prevents us from having a diagnosis of certainty of the amyloidosis subtype of each patient, therefore, it was not possible to specify what type of CA was treated in each case. In any case, given that the specificity for the diagnosis of ATTR for a grade 2 or 3 of gammagraphic uptake is $87 \%$ and that in the follow-up of patients no monoclonal gammopathy was subsequently detected, it can be stated that the vast majority of the current CA correspond to the TTR subtype. 
Secondly, the fact that no post-acquisition measurements were taken to avoid bias, meant that some parameters were not available in many patients (affecting mainly to the collection of strain values), resulting in a very low statistical power of these parameters. Nonetheless, it was considered that the fact that all the measurements were made by independent echocardiographers, without knowing the patient's diagnosis, adds value to the results that were statistically significant.

Regarding the selection of controls, slight differences in age can be attributed, but since ATTR occurs in many cases at very advanced ages, these minimal differences can be considered acceptable. In addition, parameters such as NT-proBNP and glomerular filtration that have been shown to have a high prognostic value [29] were similar. Finally, the fact that the controls emerged from the same cohort were scanned to rule out CA minimizes any selection bias that may exist.

\section{Conclusions}

In patients with LVH admitted for cardiac decompensation, a series of echocardiographic characteristics such as: the degree of LVH, left ventricular reduced size, right atrial dilatation, altered right ventricular function and strain relative apical sparing, increase the suspicion of cardiac amyloidosis and are useful for selecting candidates for performing Tc-DPD gammagraphy to establish diagnosis.

\section{Conflict of interest: None declared}

\section{References}

1. Gillmore JD, Maurer MS, Falk RH, et al. Nonbiopsy diagnosis of cardiac transthyretin amyloidosis. Circulation. 2016; 133(24): 2404-2412, doi: 10.1161/CIRCULATIONAHA.116.021612, indexed in Pubmed: 27143678.

2. Garcia-Pavia P, Domínguez F, Gonzalez-Lopez E. Amiloidosis cardíaca por transtiretina. Medicina Clínica. 2021; 156(3): 126134, doi: 10.1016/j.medcli.2020.06.064.

3. Kittleson MM, Maurer MS, Ambardekar AV, et al. Cardiac amyloidosis: evolving diagnosis and management: a scientific statement from the American Heart Association. Circulation. 2020; 142(1): e7-ee22, doi: 10.1161/CIR.0000000000000792, indexed in Pubmed: 32476490.

4. González-López E, Gallego-Delgado M, Guzzo-Merello G, et al. Wild-type transthyretin amyloidosis as a cause of heart failure with preserved ejection fraction. Eur Heart J. 2015; 36(38): 2585-2594, doi: 10.1093/eurheartj/ehv338, indexed in Pubmed: 26224076.

5. Ruberg FL. Cardiac amyloidosis: a zebra hiding in plain sight? Circ Cardiovasc Imaging. 2017; 10(3): e006186, doi: 10.1161/ CIRCIMAGING.117.006186, indexed in Pubmed: 28298289.
6. Uzan C, Lairez O, Raud-Raynier P, et al. Right ventricular longitudinal strain: a tool for diagnosis and prognosis in light-chain amyloidosis. Amyloid. 2018; 25(1): 18-25, doi: 10.1080/13506129.2017.1417121, indexed in Pubmed: 29260587.

7. Liu D, Hu K, Niemann M, et al. Effect of combined systolic and diastolic functional parameter assessment for differentiation of cardiac amyloidosis from other causes of concentric left ventricular hypertrophy. Circ Cardiovasc Imaging. 2013; 6(6): 1066-1072, doi: 10.1161/CIRCIMAGING.113.000683, indexed in Pubmed: 24100046.

8. Pagourelias ED, Mirea O, Duchenne J, et al. Echo parameters for differential diagnosis in cardiac amyloidosis: a head-to-head comparison of deformation and nondeformation parameters. Circ Cardiovasc Imaging. 2017; 10(3): e005588, doi: 10.1161/CIRCIMAGING.116.005588, indexed in Pubmed: 28298286.

9. Sun JP, Stewart WJ, Yang XS, et al. Differentiation of hypertrophic cardiomyopathy and cardiac amyloidosis from other causes of ventricular wall thickening by two-dimensional strain imaging echocardiography. Am J Cardiol. 2009; 103(3): 411-415, doi: 10.1016/j.amjcard.2008.09.102, indexed in Pubmed: 19166699.

10. Ponikowski P, Voors A, Anker S, et al. 2016 ESC Guidelines for the diagnosis and treatment of acute and chronic heart failure. The Task Force for the diagnosis and treatment of acute and chronic heart failure of the European Society of Cardiology (ESC). Developed with the special contribution of the Heart Failure Association (HFA) of the ESC. Eur J Heart Failure. 2016; 18(8): 891-975, doi: 10.1002/ejhf.592.

11. Lang RM, Badano LP, Mor-Avi V, et al. Recommendations for cardiac chamber quantification by echocardiography in adults: an update from the American Society of Echocardiography and the European Association of Cardiovascular Imaging. J Am Soc Echocardiogr. 2015; 28(1): 1-39.e14, doi: 10.1016/j. echo.2014.10.003, indexed in Pubmed: 25559473.

12. Perugini E, Guidalotti PL, Salvi F, et al. Noninvasive etiologic diagnosis of cardiac amyloidosis using 99mTc-3,3-diphosphono-1,2-propanodicarboxylic acid scintigraphy. J Am Coll Cardiol. 2005; 46(6): 1076-1084, doi: 10.1016/j.jacc.2005.05.073, indexed in Pubmed: 16168294.

13. Ruberg F, Grogan M, Hanna M, et al. Transthyretin amyloid cardiomyopathy. J Am Coll Cardiol. 2019; 73(22): 2872-2891, doi: 10.1016/j.jacc.2019.04.003.

14. Maurer MS, Schwartz JH, Gundapaneni B, et al. Tafamidis treatment for patients with transthyretin amyloid cardiomyopathy. N Engl J Med. 2018; 379(11): 1007-1016, doi: 10.1056/NEJMoa1805689, indexed in Pubmed: 30145929.

15. Adams D, Suhr OB, Dyck PJ, et al. Trial design and rationale for APOLLO, a Phase 3, placebo-controlled study of patisiran in patients with hereditary ATTR amyloidosis with polyneuropathy. BMC Neurol. 2017; 17(1): 181, doi: 10.1186/s12883-017-0948-5, indexed in Pubmed: 28893208.

16. Manolis A, Manolis A, Manolis T, et al. Cardiac amyloidosis: An underdiagnosed/underappreciated disease. Eur J Intern Med. 2019; 67: 1-13, doi: 10.1016/j.ejim.2019.07.022.

17. Maurer MS, Bokhari S, Damy T, et al. Expert consensus recommendations for the suspicion and diagnosis of transthyretin cardiac amyloidosis. Circ Heart Fail. 2019; 12(9): e006075, doi: 10.1161/CIRCHEARTFAILURE.119.006075, indexed in Pubmed: 31480867.

18. Damy T, Maurer M, Rapezzi C, et al. Clinical, ECG and echocardiographic clues to the diagnosis of TTR-related cardiomyopathy. Open Heart. 2016; 3(1): e000289, doi: 10.1136/ openhrt-2015-000289. 
19. Chacko L, Martone R, Bandera F, et al. Echocardiographic phenotype and prognosis in transthy-retin cardiac amyloidosis. Eur Heart J. 2020; 41(14): 1439-1447, doi: 10.1093/eurheartj/ehz905, indexed in Pubmed: 31950987.

20. Di Nunzio D, Recupero A, de Gregorio C, et al. Echocardiographic findings in cardiac amyloidosis: inside two-dimensional, Doppler, and strain imaging. Curr Cardiol Rep. 2019; 21(2): 7, doi: 10.1007/s11886-019-1094-z, indexed in Pubmed: 30747298.

21. Boldrini M, Cappelli F, Chacko L, et al. Multiparametric echocardiography scores for the diagnosis of cardiac amyloidosis. JACC Cardiovasc Imaging. 2020; 13(4): 909-920, doi: 10.1016/j. jcmg.2019.10.011, indexed in Pubmed: 31864973.

22. Bellavia D, Pellikka PA, Dispenzieri A, et al. Comparison of right ventricular longitudinal strain imaging, tricuspid annular plane systolic excursion, and cardiac biomarkers for early diagnosis of cardiac involvement and risk stratification in primary systematic (AL) amyloidosis: a 5-year cohort study. Eur Heart J Cardiovasc Imaging. 2012; 13(8): 680-689, doi: 10.1093/ehjci/jes009.

23. Bodez D, Ternacle J, Guellich A, et al. Prognostic value of right ventricular systolic function in cardiac amyloidosis. Amyloid. 2016; 23(3): 158-167, doi: 10.1080/13506129.2016.1194264, indexed in Pubmed: 27348696.

24. Cappelli F, Porciani MC, Bergesio F, et al. Right ventricular function in $\mathrm{AL}$ amyloidosis: characteristics and prognostic implication. Eur Heart J Cardiovasc Imaging. 2012; 13(5): 416-422, doi: 10.1093/ejechocard/jer289, indexed in Pubmed: 22180463.

25. Fitzgerald BT, Scalia GM, Cain PA, et al. Left atrial size--another differentiator for cardiac amyloidosis. Heart Lung Circ. 2011; 20(9): 574-578, doi: 10.1016/j.hlc.2011.06.002, indexed in Pubmed: 21763199.

26. Quarta CC, Solomon SD, Uraizee I, et al. Left ventricular structure and function in transthyretin-related versus light-chain cardiac amyloidosis. Circulation. 2014; 129(18): 1840-1849, doi: 10.1161/ CIRCULATIONAHA.113.006242, indexed in Pubmed: 24563469.

27. Phelan D, Collier P, Thavendiranathan P, et al. Relative apical sparing of longitudinal strain using two-dimensional speckletracking echocardiography is both sensitive and specific for the diagnosis of cardiac amyloidosis. Heart. 2012; 98(19): 1442-1448, doi: 10.1136/heartjnl-2012-302353, indexed in Pubmed: 22865865.

28. Takashio S, Yamamuro M, Izumiya Y, et al. Diagnostic utility of cardiac troponin $\mathrm{T}$ level in patients with cardiac amyloidosis. ESC Heart Fail. 2018; 5(1): 27-35, doi: 10.1002/ehf2.12203, indexed in Pubmed: 28869340.

29. Gillmore JD, Damy T, Fontana M, et al. A new staging system for cardiac transthyretin amyloidosis. Eur Heart J. 2018; 39(30): 2799-2806, doi: 10.1093/eurhearti/ehx589, indexed in Pubmed: 29048471. 